\title{
下丘脑去甲肾上腺素在饥饿对雄性大鼠 黄体生成素分泌影响中的作用
}

谢启文 刘 军

(中国医科大学, 沈阳)

我们以前的研究表明,急性饥饿时大鼠血液黄体生成素 (LH) 水平急剧降低,但垂体 LH 水平并不降低, $\mathrm{LH}$ 由循环中清除的速度及靶腺对 $\mathrm{LH}$ 的结合亦无显著改变, 提示饥饿时垂体 LH 释出减少,但其发生机理则仍末阐明 ${ }^{[1]}$. 垂体 $\mathrm{LH}$ 的分泌主要受血液中性激素水平的反错 调节和下丘脑黄体生成索释放激素 (LHRH) 的控制, 而后者本身的释出又受若干中枢递质特 别是肾上腺素能机制所调控 ${ }^{[2]}$. 为了进一步探索饥饿时 LH 释出不足的发生机理. 我们进 行了以下三方面的研究: 1. 饥饿时大鼠垂体对 LHRH 的反应，2. 饥饿时大鼠下丘脑及视前 区去甲肾上腺素 $(\mathrm{NE})$ 的更新速度, 3. 调制中枢 $\mathrm{NE}$ 递质系统对饥饿大鼠 $\mathrm{LH}$ 分䎵的影响。

\section{一、材料与方法}

全部实验均用雄性成年 Wistar 系大鼠. 急性饥饿期禁食 5 天, 但饮水不限。

1. 垂体对 LHRH 的反应 (LHRH 刺激试验) 动物在清醒、自由活动状态下,经由前 一天植人的心房留置导管 ${ }^{[3]}$ 采集第一份血样后由导管注人 LHRH (Calbiochem) $500 \mathrm{ng} / \mathrm{kg}$. 注后 30 及 $60 \mathrm{~min}$ 时分别采集第二、三份血样，然后再第二次注人 LHRH $500 \mathrm{ng} / \mathrm{kg}$. 隔 30 $\min$ 后采第四份血样. 用放射免废法测定血浆 LH 含量, 标准品为 NIADDK-rLH-RP-2.

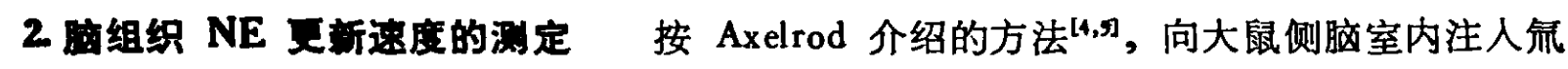
标记去甲肾上腺素 $\left(1-\left[7,8-^{-3} \mathrm{H}\right]\right.$ Noradrenaline, Amersham 产品,比放射活性 $\left.15 \mathrm{Ci} / \mathrm{mmol} / \mathrm{l}\right)$ $1.7 \mu \mathrm{Ci} / 20 \mu \mathrm{l}$. 注后 1 及 $4 \mathrm{~h}$ 分别将鼠断头, 按 Palkovitz 介绍的方法 ${ }^{[6]}$ 分别切取下丘脑和视前 区, 称重后用 $0.1 \mathrm{~mol} / 1 \mathrm{HCl}$ 制备匀浆. 离心后取上清作液闪测定. 所用仪器为 LKB Rack Beta 1215 型液闪计数仪,对 ${ }^{3} \mathrm{H}$ 的测定效率为 $60 \%$, 以外标准道比法作淬灭校正, 最后计算为 每 $\mathrm{mg}$ (湿重)脑组织的衰变计数 $(\mathrm{dpm})$. 根据 $4 \mathrm{~h}$ 与 $1 \mathrm{~h}$ 测值之差判定 $\mathrm{NE}$ 更新速度.

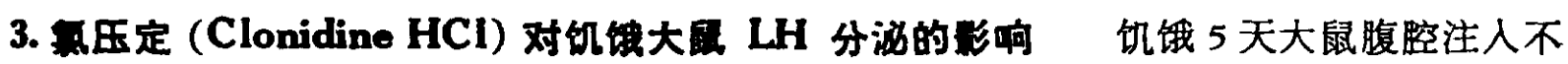
同剂量氯压定, $30 \mathrm{~min}$ 后断头, 用放免法测定血清 LH 含量, 标准品为 NIADDK-rLH-RP-1.

\section{二、结 果}

1. 急性饥馃时大垂体对 LHRH 的反应由图 1 可见,虽然饥饿鼠血液 LH 基础水 平显著低于饱食鼠者,但静脉注人 LHRH 后, 血中 $\mathrm{LH}$ 迅速升高, $30 \mathrm{~min}$ 升高的幅值与饱食

本文 1987 年 3 月 10 日收到. 
既者相同，在 $60 \mathrm{~min}$ 时第二次注入 LHRH,则血液 LH 再次迅速上升，反应强度显著超过第 一次者. $30 \mathrm{~min}$ 升高的幅度几乎为第一次反应的 3 倍. 饥饿鼠的反应仍然与饱食鼠者基本一 致，表明饥饿鼠垂体分泌 LH 的细胞对 LHRH 的敏感性或反应性并未降低。

2. 急性饥饿时大裂下丘脑和视前区 NE 更新率的改变 由表 1 可见,饱食鼠下丘脑及 视前区摄取的 ${ }^{3} \mathrm{H}-\mathrm{NE}$ 经过 $3 \mathrm{~h}$ 后均显著减少,而饥饿 5 天之大閁则否,特别是视前区, 二者的 差别尤为明显. 这表明急性饥饿时大鼠下丘脑和视前区 NE 的更新速度显著减慢。

表 1 饱食及饥饿大鼠下丘脑及视前区 NE 更新

\begin{tabular}{|c|c|c|c|c|c|c|}
\hline & & $1 \mathrm{~B}$ & & $4 \mathrm{~h}$ & & $P$ \\
\hline \multirow{2}{*}{ 下丘脑 } & 饱食 & $4128.5 \pm 483.6^{\circ}$ & $(6)^{b}$ & $2872.0 \pm 256.1$ & (7) & $<0.05$ \\
\hline & 机钱 & $4297.7 \pm 418.4$ & (8) & $3041.7 \pm 441.5$ & (6) & N.S. ${ }^{c}$ \\
\hline \multirow{2}{*}{ 规前区 } & 饱食 & $4650.2 \pm 269.2$ & (6) & $3099.6 \pm 170.9$ & (7) & $<0.01$ \\
\hline & 机锇 & $4878.2 \pm 407.3$ & (8) & $3783.1 \pm 461.4$ & (6) & N.S. \\
\hline
\end{tabular}

2. dpm/mg, $m \pm S E ;$ b. 例数; c. 不显著.

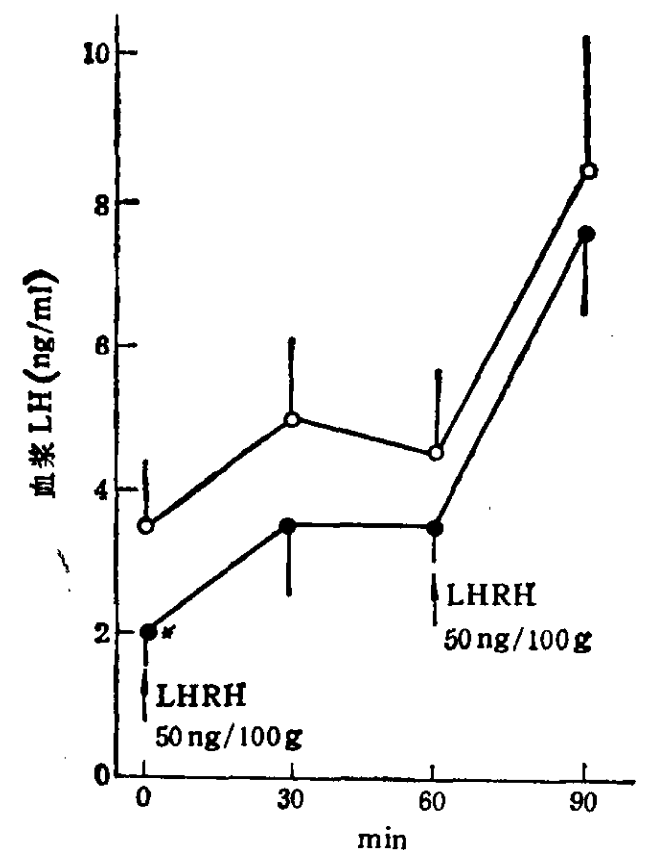

图 1 饱食及饥饿大閖垂体(生体内) 对 LHRH 的 LH 释出反应

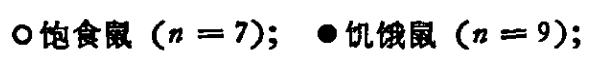
*与饱食鼠差别显著

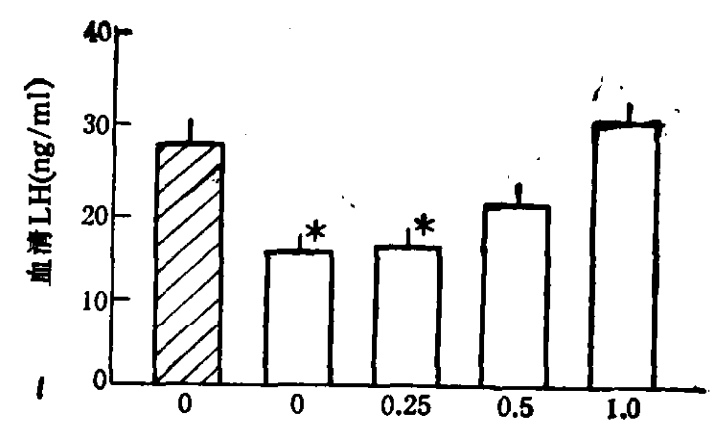

聚压定新 $\mathbf{2}(\mathrm{mg} / \mathrm{kg})$

图 2 昰压定对饥饿大鼠 LH 分班的影响

四脃食鼠；口机钱闵； *与垉食鼠差别显著

3. 压定对急性饥饿大鼠 LH 分泌的影响 由图 2 可见, $\alpha$-去甲肾上腺素能激动剂" 彔压定对饥饿鼠 LH 分泌有明显的剌激作用, 并呈剂量-效应关系. $0.25 \mathrm{mg} / \mathrm{kg}$ 以下效果不. 明显, $1 \mathrm{mg} / \mathrm{kg}$ 则使血 $\mathrm{LH}$ 水平升高到饱食鼠的范围.

\section{三、讨论}

垂体 LH 的分泌一方面取决于下丘脑 LHRH 的释放量和垂体 LH-分脑细胞对 LHRH 
的敏感性,另一方面受血中性激素水平的反馈性调节. 本文全部使用雄性大鼠,避免血液性激 索水平周期性波动的影响. 因此饥饿时垂体 LH 分泌减少, 从原则上考虑, 主要可能是 $L H R H$ 的释出不足或/及垂体对 LHRH 敏感性降低. 迄今尚未见有关于饥饿时垂体门脉血中 LHRH 含量测定的报告. 从本文结果分析, 饥饿时垂体对 LHRH 敏感性降低的可能性不大. 因为饥 饿鼠垂体受 LHRH 作用时, 其 LH 分必反应, 不论速度或幅度都不亚于饱食鼠者. 特别是在 LHRH 调控 LH 分泌中有重要作用的预敏效应 (priming effect), 即预先的 LHRH 作用将 增强继后的 LHRH 作用所引起的反应, 在饥饿时亦未减弱. 因此可以认为急性饥饿时 LH 释出减少. 主要不是由于垂体对 LHRH 繁感性降低,而是由于下丘脑 LHRH 释出不足. 在 本实验中虽然没有直接测定下丘脑的 LHRH 含量, 但 Badger 等报告急性饥饿鼠垂体细胞 在生物体外对 LHRH 反应正常 ${ }^{[7]}$; Pirke 与 Spyra 报告饥饿大鼠正中隆起 LHRH 静态含 量测定比饱食鼠高 ${ }^{\text {[e }}$, 都符合上述推断。

近年来的研究表明, LHRH 神经元的细胞体主要在视前区-前部下丘脑和内侧基底下丘 脑 (弓状核), 前者与 LH 的周期性分修有较密切的关系. 去甲肾上腺素能神经终末与 LHRH 神经元发生突触连系, 通过其递质 NE 控制(主要是刺激) LHRH 的释出 ${ }^{[2,91}$. 因此研究这两 个脑区 NE 的更新状况对阐明 LHRH 释出减少的机理是有意义的.

Glowinski 等报告, 大鼠侧脑室注人的 ${ }^{3} \mathrm{H}-\mathrm{NE}$ 迅即被 NE 神经终末摄取 (1h 达最高) 并

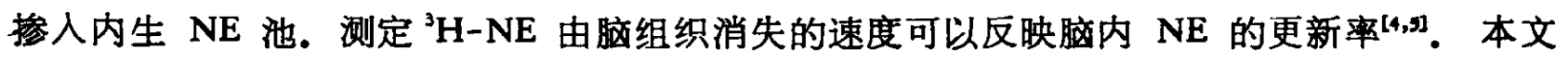
应用这一技术证明, 急性饥饿 5 天后大鼠下丘脑, 特别是视前区, $\mathrm{NE}$ 更新速度显著减慢。另 一方面， $\boldsymbol{\alpha}$-去甲肾上腺素能激动剂氯压定则能使饥饿鼠 LH 分泌增吅, 呈剂量-效应关系. $1 \mathrm{mg} / \mathrm{kg}$ 时可使饥饿鼠血 LH 升高到正常范围. 这些结果表明肾上腺素能机制紊乱在急性饥 饿时 LH 分泌减少的发生中有重要作用. 特别令人感兴趣的是,最近我们发现: 慢性饥饿使 倠鼠的动情周期严重紊乱, 甚至停止. 但如在饥饿期间投与氯压定, 则在相当程度上防止了饥 饿的这一影响(待发表的资料),进一步支持了我们的论点.

本文主要分析去甲肾上腺素的作用, 但调控 LHRH 释出的机理比较复杂, 除 NE 外还 有其它递质的参与. 例如已有证据表明下丘脑结节漏斗多巴胺 (TIDA) 神经元参与抑制 LHRH 的释放 ${ }^{[2]}$. 著者过去的研究已证明在急性饥饿时这一递质系统受到激活, 多巴胺释出 增多 ${ }^{[00]}$. 这也可能是急性饥饿时 LHRH 释出减少的又一个因素.

致谢: 本文所用大鼠 LH 敒免测定药盒由 NIADDK 提供,特此致谢.

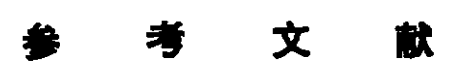

[1] 谢启文、刘军,科学通报, 32(1987), 7: 545 .

[2] 谢启文,生殖与避肎, 5(1985), 3: 12.

[3] 谢启文,中国医科大学学报, 13(1984), 4: 73.

[ 4 ] Glowinski, J. \& Axelrod, J., J. Pharmacol. Exp. Ther, 149(1965), 43.

[ 5 ] Glowinski, J. et ai., J. Neurochem., 12(1965), 25.

[ 6 ] Palkovitz, M., Int. Rev. Cyzol., 56(1979), 315.

[ 7 B Badger, T. M. et al., J. Nutr., 115(1985), 788.

[ 8 ] Pirke, K. M. \& Spyra, B., Acta Endocr., 96(1981), 413.

[ 9 ] Adler, N. T. (ed.), Neuroendoc rinology of Reproduction, Plenum Press, New York, 1981, 444

[10] 驸启文, 科学通报, 29(1984), 8: 567 . 